sie beherrschen die Werkzeuge für deren Auswertung. Darüber hinaus braucht es aber auch interessierte Journalisten, die die relevanten Fragen stellen, Visualisierungs-Ideen einbringen und das Publikum anlocken.

Was kann aus solchen Kooperationen herauskommen? Etwas Eindrucksvolles, wie an einem gemeinsamen Projekt der Tageszeitung „Die Welt" und der HafenCity Universität Hamburg zu erkennen ist. Johannes Kröger von der HCU stellte das Ergebnis vor: eine Webreportage zur Elbvertiefung. Er berichtete, wie die lokale Redaktion der „Welt“ die Idee an den Campus brachte: „Wir möchten gern die Elbe einmal unter Wasser zeigen.“

Das Problem dabei: Wie lassen sich für so ein großes Gebiet wie der gesamte Elbverlauf kleinste Details zeigen? Die Lösung war eine Kombination aus Video und 3D-Viewer. Das Video, um die Elbe einmal komplett abzufliegen, der 3D-Viewer um Details auf dem Flussgrund wie beispielsweise Wellenstrukturen, den alten Elbtunnel und Schiffwracks erkunden zu können. Der Lohn der Mühe: Neben vielen positiven Rückmeldungen aus der Öffentlichkeit wurde das Team für den Reporterpreis 2016 nominiert.

Was gab es noch zu entdecken? Mathias Gröbe von der TU Dresden stellte Techniken, Workflows und Darstellungsformen zur Analyse von Social-Media-Con-

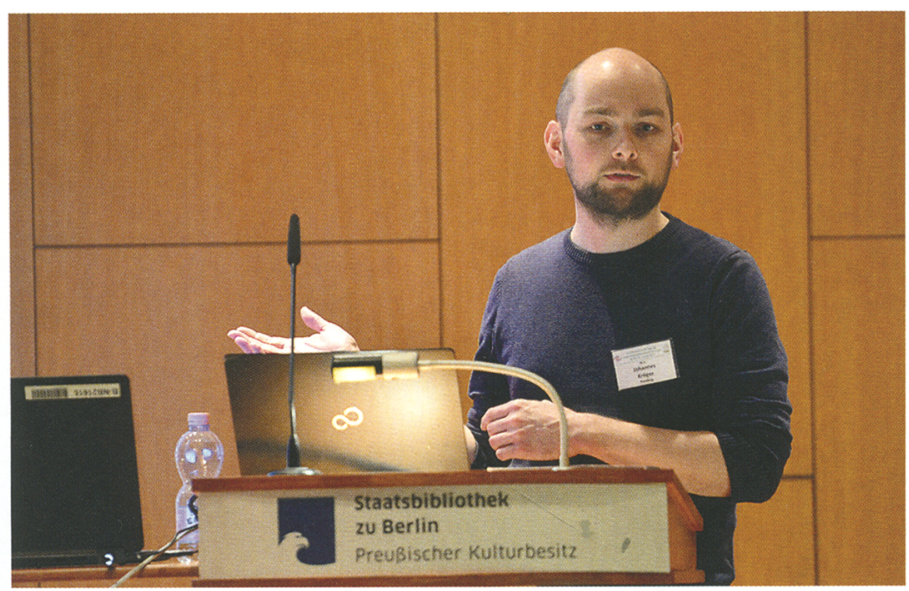

Abb. 3: Referent Johannes Kröger von der HCU. Foto: dpa 2017 tent vor. Deutlich wurde dabei, dass neben den technischen Herausforderungen vor allem die statistische Signifikanz noch Probleme bereitet. Datenjournalisten der „Berliner Morgenpost“ und Studenten der HafenCity Universität beschäftigten sich im Rahmen des Projekts CoGran mit der Frage, wie sich Statistiken verschiedener geographischer Bezugsräume vereinheitlichen lassen. Inga Schlegel von der HCU erklärte das viel diskutierte Tool und zeigte Anwendungsbeispiele wie eine Berlin-Wahlkarte. Bei der schnellen Produktion von Online-Karten für das aktuelle Nachrichtengeschehen sieht der Entwickler Lorenz Matzat noch Potenzial und präsentierte sein Kartentool mapworks.

Was bei den Diskussionen nach den Beiträgen, aber auch bei der anschließenden Diskussion zu Synergien zwischen Journalismus und Kartographie auffiel: Die Tagungsteilnehmer wissen oft noch wenig von der Profession, dem Arbeitsalltag der jeweils anderen und haben Mühe, die fremde (Fach-)Sprache zu übersetzen. Doch Raimar Heber, einer der beiden Gastgeber, formulierte treffend: „Die Verzahnung ist noch relativ zaghaft, aber ein Anfang ist gemacht."

Sebastian Tanke, dpa infografik, Berlin

Weitere Berichte auch auf: http:// news-infographics-maps.net

\title{
Horst Schöttler erhält THW-Ehrenzeichen in Gold
}

\section{Besondere Auszeichnung für DGfK-Ehrenmitglied}

Dr. Horst Schöttler, langjähriges und sehr engagiertes Mitglied der DGfK, Vorstandsvorsitzender der Kartographie-Stiftung Ravenstein, hat eine besondere Ehrung am 14. Januar 2017 erhalten. Der Ehrenpreisträger hat sich über viele Jahre für das THW, insbesondere für den Katastrophenschutz und die zivile Verteidi- Mark Vetter, Karlsruhe

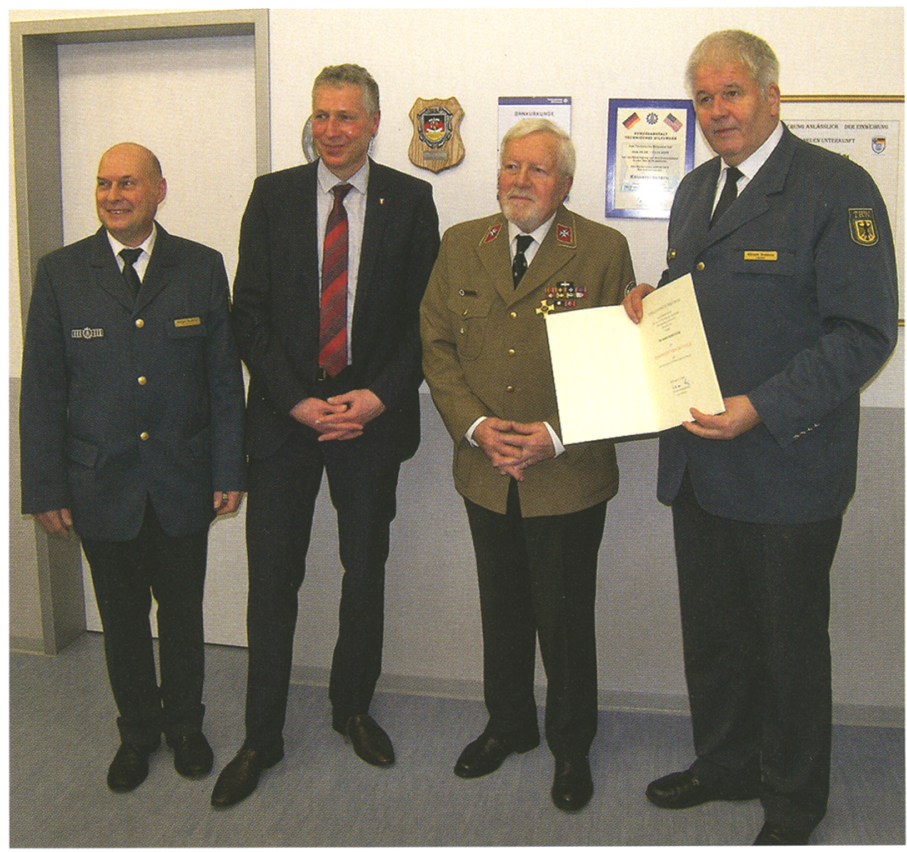

Bei der Urkundenverleihung: V. li. stv. OB des THW Kaiserslautern, Dipl.-Ing. Rüdiger Hemberle, Beigeordneter Peter Kiefer, Feuerwehr- und KatS-Dezernent der Stadt Kaiserslautern, Dr. Horst Schöttler, Dipl.-Ing. Albrecht Broemme, Präsident der Bundesanstalt THW. Foto: Matthias Nickel

\section{Langjährige DGfK-Mitgliedschaft März/April 2017}

Folgenden Mitgliedern dankt die Deutsche Gesellschaft für Kartographie für ihre langjährige Treue:

\section{Mitglied seit 60 Jahren:}

Dipl.-Ing. (FH) Helmut Schaub, 70567 Stuttgart

\section{- Mitglied seit 50 Jahren:}

Werner Brandenstein,

48163 Münster

Dipl.-Ing. (FH) Hans Hofmann, 81243 München

Dipl.-Ing. (FH) Klaus Logge, 13469 Berlin
Prof. Dr. Bernd Meissner, 13503 Berlin

Prof. Dipl.-Ing. Peter Mellmann, 82223 Eichenau

Prof. Dr. Siegmund Schulz, 12209 Berlin

Dipl.-Ing. (FH) Helmut Uhrig, 97840 Hafenlohr-Windheim

Mitglied seit 40 Jahren:

Dipl.-Ing. Ute Hausen, 53879 Euskirchen

Dipl.-Ing. (FH) Eckhard Meier-Wölfle, 55283 Nierstein

Dipl.-Ing. (FH) Reinhold Miklis, 12683 Berlin

Dipl.-Ing. Sabine Urbanke, 71394 Kernen-Stetten 